\title{
On Existence and Multiplicity of Solutions for Elliptic Equations Involving the $p$-Laplacian
}

Xian $\mathrm{Wu}$ and Jilin Chen

Abstract. In the present paper, the following Dirichlet problem and Neumann problem involving the $p$-Laplacian

$$
\left\{\begin{array}{l}
-\Delta_{p}(u)=\lambda f(x, u), \quad \text { in } \Omega \\
u=0, \text { on } \partial \Omega
\end{array}\right.
$$

and

$$
\left\{\begin{array}{l}
-\Delta_{p}(u)+a(x)|u|^{p-2} u=\lambda f(x, u), \quad \text { in } \Omega \\
\frac{\partial u}{\partial n}=0, \quad \text { on } \partial \Omega
\end{array}\right.
$$

are studied and some new multiplicity results of solutions for systems $(1 . \lambda)$ and $(2 . \lambda)$ are obtained. Moreover, by using the KKM principle we give also two new existence results of solutions for systems (1.1) and (2.1).

Mathematics Subject Classification (2000). 35J20, 35J70, 35P05, 35P30,34B15, 58E05, 47H04.

Keywords. Critical point, $p$-Laplacian, Sobolev's embedding, Dirichlet problem, Neumann problem.

\section{Introduction and preliminaries}

Consider the following Dirichlet problem and Neumann problem involving the $p$ Laplacian

$$
\left\{\begin{array}{l}
-\Delta_{p}(u)=\lambda f(x, u), \text { in } \Omega \\
u=0, \text { on } \partial \Omega
\end{array}\right.
$$

and

$$
\left\{\begin{array}{l}
-\Delta_{p}(u)+a(x)|u|^{p-2} u=\lambda f(x, u), \quad \text { in } \quad \Omega \\
\frac{\partial u}{\partial n}=0, \quad \text { on } \partial \Omega
\end{array}\right.
$$

where $\Omega \subset R^{N}$ is a bounded domain, $a \in L^{\infty}(\Omega)$ with essinf $\operatorname{in}_{\Omega} a>0, N \geq 1$, $p>1, \lambda>0$ and $f: \Omega \times R \rightarrow R$ is a Carathéodory function, that is, $f(x, t)$ is measurable in $x$ for every $t \in R$ and continuous in $t$ for a.e. $x \in \Omega$. Here, $\Delta_{p}$ is 
the $p$-Laplacian, i.e. $\Delta_{p}(u)=\operatorname{div}\left(|\nabla u|^{p-2} \nabla u\right)$. Let $\lambda_{1}=\min \left\{\int_{\Omega}|\nabla u|^{p} d x: u \in\right.$ $\left.W_{0}^{1, p}(\Omega), \int_{\Omega} V|u|^{p} d x=1\right\}$ is the first eigenvalue of the problem

$$
\left\{\begin{array}{l}
-\Delta_{p}(u)=\lambda V(x)|u|^{p-2} u, \text { in } \Omega \\
u=0, \text { on } \partial \Omega .
\end{array}\right.
$$

where $V \in L^{s}(\Omega)$ for some $s>\frac{N}{p}$ if $1<p \leq N$ and $s=1$ if $p>N$. Recall that $\lambda_{1}$ is simple, positive and isolated (see [4]). Moreover, there exists a unique positive eigenfunction $w_{1}$ whose norm in $W_{0}^{1, p}(\Omega)$ is 1 and $W_{0}^{1, p}(\Omega)=E_{1} \oplus E_{2}$, where $E_{1}=\operatorname{span}\left\{w_{1}\right\}, E_{2}=\left\{u \in W_{0}^{1, p}(\Omega): \int_{\Omega} V u w_{1}^{p-1} d x=0\right\}$ and the following proposition holds:

Proposition 1. There exists a number $\bar{\lambda}>\lambda_{1}$ such that

$$
\int_{\Omega}|\nabla u|^{p} d x \geq \bar{\lambda} \int_{\Omega} V|u|^{p} d x
$$

for all $u \in E_{2}$.

Proof. Assume by contradiction that there exisis a sequence $\left\{u_{n}\right\}$ in $E_{2}$ such that

$$
\int_{\Omega}\left|\nabla u_{n}\right|^{p} d x<\left(\lambda_{1}+\frac{1}{n}\right) \int_{\Omega} V\left|u_{n}\right|^{p} d x
$$

for all $n \in\{1,2,3, \ldots\}$. Let $v_{n}=\frac{u_{n}}{\left(\int_{\Omega} V\left|u_{n}\right|^{p} d x\right)^{\frac{1}{p}}}$. Then $\int_{\Omega} V\left|v_{n}\right|^{p} d x=1$ and $\left\|v_{n}\right\|^{p}<\lambda_{1}+\frac{1}{n}$. Hence $\left\{v_{n}\right\}$ is bounded in $W_{0}^{1, p}(\Omega)$. Due to the reflexivity of $W_{0}^{1, p}(\Omega)$ and the compact embedding $W_{0}^{1, p}(\Omega) \hookrightarrow L^{p}(\Omega)$, we can assume that there exists $v \in W_{0}^{1, p}(\Omega)$ such that

$$
v_{n} \rightarrow v \quad(\text { i.e. weakly }) \text { in } W_{0}^{1, p}(\Omega),
$$

and

$$
v_{n} \rightarrow v \quad \text { (i.e. strongly) in } L^{p}(\Omega) \text {. }
$$

Hence $\int_{\Omega} V|v|^{p} d x=1$ and so

$$
\lambda_{1} \leq \int_{\Omega}|\nabla v|^{p} d x \leq \liminf _{n \rightarrow \infty} \int_{\Omega}\left|\nabla v_{n}\right|^{p} d x \leq \limsup _{n \rightarrow \infty} \int_{\Omega}\left|\nabla v_{n}\right|^{p} d x \leq \lambda_{1} .
$$

Therefore, $\left\|v_{n}\right\| \rightarrow\|v\|$ and $\int_{\Omega}|\nabla v|^{p} d x=\lambda_{1}$. This implies that $v$ is the first eigenfunction, i.e. $v \in E_{1}$. On the otherhand, since each $v_{n} \in E_{2}$,

$$
\int_{\Omega} V v_{n} w_{1}^{p-1} d x=0, \quad \forall n
$$

Note that $v_{n} \rightarrow v$ in $W_{0}^{1, p}(\Omega)$. Hence $\int_{\Omega} V v w_{1}^{p-1} d x=0$, i.e. $v \in E_{2}$. This shows $v=0$. It contradicts the fact that $\int_{\Omega} V|v|^{p} d x=1$. This completes the proof.

The energy functionals associated to problems $(1 . \lambda)$ and $(2 . \lambda)$, namely $\varphi_{1 \lambda}$ and $\varphi_{2 \lambda}$, on $W_{0}^{1, p}(\Omega)$ and $W^{1, p}(\Omega)$ given by, respectively,

$$
\varphi_{1 \lambda}(u)=\frac{1}{p}\|u\|^{p}-\lambda \int_{\Omega} F(x, u) d x
$$


and

$$
\varphi_{2 \lambda}(u)=\frac{1}{p}\|u\|_{0}^{p}-\lambda \int_{\Omega} F(x, u) d x
$$

are differentiable and

$$
\left\langle\varphi_{1 \lambda}^{\prime}(u), v\right\rangle=\int_{\Omega}|\nabla u|^{p-2} \nabla u \nabla v d x-\lambda \int_{\Omega} f(x, u) v d x
$$

for $u, v \in W_{0}^{1, p}(\Omega)$,

$$
\left\langle\varphi_{2 \lambda}^{\prime}(u), v\right\rangle=\int_{\Omega}\left[|\nabla u|^{p-2} \nabla u \nabla v+a(x)|u|^{p-2} u v\right] d x-\lambda \int_{\Omega} f(x, u) v d x
$$

for $u, v \in W^{1, p}(\Omega)$, where $F(x, t)=\int_{0}^{t} f(x, s) d s,\|u\|=\left(\int_{\Omega}|\nabla u|^{p} d x\right)^{\frac{1}{p}}$ and $\|u\|_{0}=$ $\left(\int_{\Omega}\left[|\nabla u|^{p}+a(x)|u|^{p}\right] d x\right)^{\frac{1}{p}}$. It is well known that the weak solutions of the problems $(1 . \lambda)$ and $(2 . \lambda)$ correspond to the critical points of $\varphi_{1 \lambda}$ and $\varphi_{2 \lambda}$, respectively. As $\lambda=1$ we denote $\varphi_{1 \lambda}=\varphi_{1}$ and $\varphi_{2 \lambda}=\varphi_{2}$.

In recent papers [2] and [3], problems $(2 . \lambda)$ and $(1 . \lambda)$ were researched and the following two multiplicity theorems were given, respectively.

Theorem A. Assume that $p>N, f$ is such that, for each $r>0, \sup _{|t| \leq r}|f(x, t)| \in$ $L^{1}(\Omega)$, and assume that there exist three positive constants $\gamma, \delta$ and $s$, with $\gamma<\delta$ and $s<p$ and a function $\mu \in L^{1}(\Omega)$ such that

(i) $\int_{\Omega} \sup _{t \in[-\gamma, \gamma]} F(x, t) d x<\frac{1}{k^{p}\|a\|_{1}}\left(\frac{\gamma}{\delta}\right)^{p} \int_{\Omega} F(x, \delta) d x$, where $k=\sup _{u \in W^{1, p}(\Omega) \backslash\{0\}} \frac{\|u\|_{\infty}}{\|u\|_{0}}$,

(ii) $F(x, t) \leq \mu(x)\left(1+|t|^{s}\right)$ for all $t \in R$ and a.e. $x \in \Omega$.

Then there exists an open interval $\Lambda \subset[0,+\infty)$ and a positive real number $\rho$ such

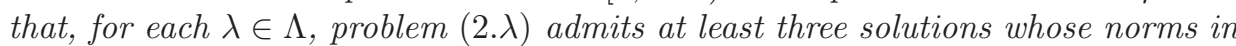
$W^{1, p}(\Omega)$ are less than $\rho$.

Theorem B. Assume that $p>N, f$ is such that, for each $s>0, \sup _{|t| \leq s}|f(x, t)| \in$ $L^{1}(\Omega)$, and assume that there exist two positive constants $c, \gamma$ with $\gamma<p, a \in$ $L^{1}(\Omega)$ and $u^{*} \in W_{0}^{1, p}(\Omega)$ such that

(i) $\left\|u^{*}\right\|>\frac{c}{k}$, where $k=\sup _{u \in W_{0}^{1, p}(\Omega) \backslash\{0\}} \frac{\max _{x \in \bar{\Omega}}|u(x)|}{\|u\|}$,

(ii) $\frac{1}{c^{p}} \int_{\Omega} \sup _{t \in[-c, c]} F(x, t) d x<\frac{1}{k^{p}} \frac{\int_{\Omega} F\left(x, u^{*}(x)\right) d x}{\left\|u^{*}\right\|^{p}}$,

(iii) $F(x, t) \leq a(x)\left(1+|t|^{\gamma}\right)$ for all $t \in R$ and a.e. $x \in \Omega$.

Then there exists an open interval $\Lambda \subset[0,+\infty)$ and a positive real number $\rho$ such

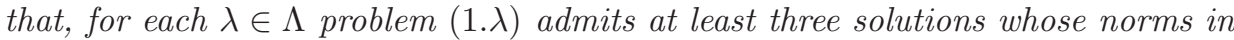
$W_{0}^{1, p}(\Omega)$ are less than $\rho$.

In the present paper, our main purposes are to give some new multiplicity

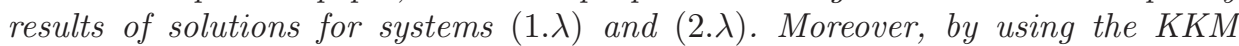
principle we give also some new existence results of solutions for systems (1.1) and (2.1). 


\section{Main results}

Let us begin by considering the general case.

Theorem 1. Assume that $p>N$ and $f$ is such that, for each $s>0, \sup _{|t| \leq s}|f(x, t)| \in$ $L^{1}(\Omega)$. If the following conditions hold:

(i) there exist $g, h \in L^{1}(\Omega, R), M>0$ and $\alpha \in[0, p-1)$ such that

$$
\frac{f(x, t)}{t} \leq \frac{g(x)|t|^{\alpha-1} t+h(x)}{t}
$$

for all $|t| \geq M$ and a.e. $x \in \Omega$,

(ii) there exist two numbers $c>0$ and $\delta>0$ with $c<\delta$ such that

$$
\int_{\Omega} \sup _{t \in[-c, c]} F(x, t) d x<\frac{1}{k^{p}\|a\|_{1}}\left(\frac{c}{\delta}\right)^{p} \int_{\Omega} F(x, \delta) d x
$$

where $k=\sup _{u \in W^{1, p}(\Omega) \backslash\{0\}} \frac{\|u\|_{\infty}}{\|u\|_{0}}$, then there exists an open interval $\Lambda \subset$ $[0,+\infty)$ and a positive real number $\rho$ such that, for each $\lambda \in \Lambda$, problem (2. $\lambda)$ admits at least three solutions whose norms in $W^{1, p}(\Omega)$ are less than $\rho$.

Proof. Let

$$
\Phi(u)=\frac{\|u\|_{0}^{p}}{p}, \quad \Psi(u)=-\int_{\Omega} F(x, u) d x
$$

for all $u \in W^{1, p}(\Omega)$. By the weakly lower semicontinuity of norm we know that $\Phi$ is weakly lower semicontinuous on $W^{1, p}(\Omega)$. Since $p>N$, the embedding operator $i: W^{1, p}(\Omega) \hookrightarrow C^{0}(\Omega)$ is compact. Hence $0<k<+\infty$ and $\Psi$ are weakly lower semicontinuous on $W^{1, p}(\Omega)$. Indeed, if the sequence $\left\{u_{n}\right\} \subset W^{1, p}(\Omega)$ with $u_{n} \rightarrow u$, then we may assume that $u_{n} \rightarrow u$ in $C^{0}(\Omega)$. We need to prove that $\Psi(u) \leq$ $\sup _{m} \inf _{n>m} \Psi\left(u_{n}\right)$. If this is false, then there exist $\varepsilon_{0}>0$ and a subsequence $\left\{u_{n_{k}}\right\}$ such that $\Psi(u)-\Psi\left(u_{n_{k}}\right)>\varepsilon_{0}$. Note that $\sup _{|t| \leq s}|f(x, t)| \in L^{1}(\Omega)$ for each $s>0$. By Lebesgue control convergence theorem we know that

$$
0=\lim _{k \rightarrow \infty}\left[\Psi(u)-\Psi\left(u_{n_{k}}\right)\right] \geq \varepsilon_{0},
$$

a contradiction. This shows that $\Psi$ is weakly lower semicontinuous on $W^{1, p}(\Omega)$.

Now, we prove that, for each $\lambda>0$,

$$
\lim _{\|u\| \rightarrow \infty}(\Phi(u)+\lambda \Psi(u))=+\infty .
$$

Indeed, let $\delta(x, t)=f(x, t)-g(x)|t|^{\alpha-1} t-h(x)$ and

$$
\beta(x)=\max \left\{\sup _{0 \leq t<M} \delta(x, t),-\inf _{-M<t \leq 0} \delta(x, t)\right\} .
$$

Then

$$
\delta(x, t) t \leq \begin{cases}0, & \text { if } \quad|t| \geq M \\ \beta(x)|t|, & \text { if } \quad|t|<M\end{cases}
$$


for a.e. $x \in \Omega$. Hence

$$
\delta(x, s t) t \leq \begin{cases}0, & \text { if } \quad s \geq \frac{M}{|t|} \\ \beta(x)|t|, & \text { if } \quad 0<s<\frac{M}{|t|} .\end{cases}
$$

Consequently,

$$
\begin{aligned}
F(x, t) & =\int_{0}^{t} f(x, s) d s \\
& =\int_{0}^{1} f(x, t s) t d s \\
& =\int_{0}^{1} \delta(x, t s) t d s+\int_{0}^{1} g(x)|t|^{\alpha+1} s^{\alpha} d s+\int_{0}^{1} h(x) t d s \\
& \leq \beta(x) M+\frac{1}{\alpha+1} g(x)|t|^{\alpha+1}+h(x) t
\end{aligned}
$$

and so that

$$
\begin{aligned}
\int_{\Omega} F(x, u) d x & \leq \int_{\Omega}\left[\beta(x) M+\frac{1}{\alpha+1} g(x) u^{\alpha+1}+h(x) u\right] d x \\
& \leq M \int_{\Omega}|\beta(x)| d x+\frac{1}{\alpha+1} \int_{\Omega}|g(x) \| u|^{\alpha+1} d x+\int_{\Omega}|h(x)||u| d x \\
& \leq M \int_{\Omega}|\beta(x)| d x+\frac{1}{\alpha+1}\|u\|_{\infty}^{\alpha+1} \int_{\Omega}|g(x)| d x+\|u\|_{\infty} \int_{\Omega}|h(x)| d x \\
& \leq c_{1}+c_{2}\|u\|_{0}^{\alpha+1}+c_{3}\|u\|_{0} .
\end{aligned}
$$

Therefore, for each $u \in W^{1, p}(\Omega)$ and each $\lambda>0$, we have

$$
\begin{aligned}
\Phi(u)+\lambda \Psi(u) & =\frac{1}{p}\|u\|_{0}^{p}-\lambda \int_{\Omega} F(x, u) d x \\
& \geq \frac{1}{p}\|u\|_{0}^{p}-\lambda\left[c_{1}+c_{2}\|u\|_{0}^{\alpha+1}+c_{3}\|u\|_{0}\right] .
\end{aligned}
$$

Since $0 \leq \alpha<p-1$,

$$
\lim _{\|u\|_{0} \rightarrow+\infty}[\Phi(u)+\lambda \Psi(u)]=+\infty .
$$

The rest parts of the proof are same with Theorem 2 in [2].

Remark. There are functions satisfying Theorem 1 and not satisfying Theorem A.

Example. Consider the following problem

$$
\left\{\begin{array}{l}
-\operatorname{div}(|\nabla u| \nabla u)+\frac{2+x}{\pi}|u| u=\lambda\left(x^{2}+y^{2}\right) \beta(u), \quad \text { in } \Omega \\
\frac{\partial u}{\partial n}=0, \text { on } \partial \Omega
\end{array}\right.
$$

where $\Omega=\left\{(x, y) \in R^{2}: x^{2}+y^{2} \leq 1\right\}$ and

$$
\beta(t)=\left\{\begin{array}{lll}
2 e^{-t^{2}} t^{17}\left(9-t^{2}\right), & \text { if } & |t| \leq 2, \\
85 \times 2^{13} \times e^{-4} \frac{4 t^{3}}{1+t^{4}}, & \text { if } & |t| \geq 2 .
\end{array}\right.
$$


In Theorem 1, we take $N=2, p=3, \alpha=1, c=1, \delta=2$ and $f(x, y, t)=\left(x^{2}+\right.$ $\left.y^{2}\right) \beta(t), a(x, y)=\frac{2+x}{\pi}$ for all $(x, y) \in \Omega$ and $t \in R$. Then, all the assumptions of Theorem 1 are satified, but the condition (ii) of Theorem A is not satisfied.

Indeed, $\|a\|_{1}=2,\|a\|_{\infty}=\frac{3}{\pi}, \int_{\Omega} \sup _{|t| \leq 1} F(x, y, t) d x d y=\frac{\pi}{2 e}, \int_{\Omega} F(x, y, \delta) d x d y=$ $2^{17} \times e^{-4} \pi$ and by Remark 1 in [2] we know that $k^{3}\|a\|_{1} \leq \frac{16 \times 27}{\pi}$. Hence the condition (ii) of Theorem 1 holds. Moreover, as $M$ is enough large the condition (i) of Theorem 1 holds, too. But, the condition (ii) of Theorem A is not satisfied, obviously.

Theorem 2. Assume that $p>N$ and $f$ is such that, for each $s>0, \sup _{|t| \leq s}|f(x, t)| \in$ $L^{1}(\Omega)$. If the following conditions hold:

(i) there exist $g, h \in L^{1}(\Omega, R), M>0$, and $\alpha \in[0, p-1)$ such that

$$
\frac{f(x, t)}{t} \leq \frac{g(x)|t|^{\alpha-1} t+h(x)}{t}
$$

for all $|t| \geq M$ and a.e. $x \in \Omega$,

(ii) there exist a number $c>0$ and an $u^{*} \in W_{0}^{1, p}(\Omega)$ such that

(a) $\left\|u^{*}\right\|>\frac{c}{k}$, where

$$
k=\sup _{u \in W_{0}^{1, p}(\Omega) \backslash\{0\}} \frac{\max _{x \in \bar{\Omega}}|u(x)|}{\|u\|},
$$

(b) $\frac{1}{c^{p}} \int_{\Omega} \sup _{t \in[-c, c]} F(x, t) d x<\frac{1}{k^{p}} \frac{\int_{\Omega} F\left(x, u^{*}(x)\right) d x}{\left\|u^{*}\right\|^{p}}$,

then there exists an open interval $\Lambda \subset[0,+\infty)$ and a positive real number $\rho$

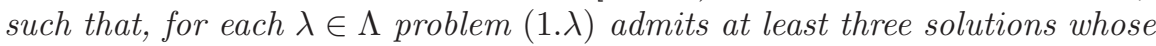
norms in $W_{0}^{1, p}(\Omega)$ are less than $\rho$.

Proof. Let

$$
\Phi(u)=\frac{\|u\|^{p}}{p}, \quad \Psi(u)=-\int_{\Omega} F(x, u) d x
$$

for all $u \in W_{0}^{1, p}(\Omega)$. The rest parts of the proof are similar to Theorem 1 .

Now, we consider the case that $\lambda=1$. We have the following existence and multiplicity theorems.

As a new research method for the existence of solutions, we use KKM principle (see [5]) to prove the following Theorem 3. For this reason, we give the concept of KKM mapping. Set $E$ be a linear space, $X$ a nonempty subset of $E$ and $G: X \rightarrow 2^{E}$ a multivalued mapping. $G$ is said be a KKM mapping if for any finite set $\left\{x_{1}, x_{2}, \ldots, x_{n}\right\} \subset X$, one has

$$
\operatorname{co}\left\{x_{1}, x_{2}, \ldots, x_{n}\right\} \subset \bigcup_{i=1}^{n} G\left(x_{i}\right) .
$$

Theorem 3. If there exist $\alpha \in[0, p-1), g \in L^{\frac{p}{p-\alpha-1}}\left(\Omega, R^{+}\right)$and $h \in L^{q}\left(\Omega, R^{+}\right)$ $\left(q=\frac{p}{p-1}\right)$ such that

$$
|f(x, t)| \leq g(x)|t|^{\alpha}+h(x)
$$


for every $t \in R$ and a.e. $x \in \Omega$, then problem (2.1) has at least a solution in $W^{1, p}(\Omega)$.

Proof. For each $v \in W^{1, p}(\Omega)$, let

$$
P(v)=\left\{u \in W^{1, p}(\Omega):\left\langle\varphi_{2}^{\prime}(u), v-u\right\rangle \geq 0\right\} .
$$

Then $P: W^{1, p}(\Omega) \longrightarrow 2^{W^{1, p}(\Omega)}$ is a KKM mapping. Indeed, for each finite subset $\left\{v_{1}, v_{2}, \ldots, v_{n}\right\}$ of $W^{1, p}(\Omega)$, if there exists a point $u_{0} \in \operatorname{co}\left\{v_{1}, v_{2}, \ldots, v_{n}\right\}$ such that $u_{0} \notin \bigcup_{i=1}^{n} P\left(v_{i}\right)$, then

$$
\left\langle\varphi_{2}^{\prime}\left(u_{0}\right), v_{i}-u_{0}\right\rangle<0, \quad i=1,2, \ldots, n .
$$

Let $u_{0}=\sum_{i=1}^{n} \alpha_{i} v_{i}$, where each $\alpha_{i} \geq 0$ and $\sum_{i=1}^{n} \alpha_{i}=1$. Then

$$
0=\left\langle\varphi_{2}^{\prime}\left(u_{0}\right), u_{0}-u_{0}\right\rangle=\sum_{i=1}^{n} \alpha_{i}\left\langle\varphi_{2}^{\prime}\left(u_{0}\right), v_{i}-u_{0}\right\rangle<0 .
$$

This is a contradiction. Hence $P: W^{1, p}(\Omega) \longrightarrow 2^{W^{1, p}(\Omega)}$ is a KKM mapping.

Moreover, for each $v \in W^{1, p}(\Omega), P(v)$ is a weakly compact subset of $W^{1, p}(\Omega)$. In fact, note that $\alpha \in[0, p-1)$ and ess $\inf _{\Omega} a>0$, by Hölder inequality and the definition of $\|\cdot\|_{0}$ we have

$$
\begin{aligned}
\left\langle\varphi_{2}^{\prime}(u), v-u\right\rangle= & \int_{\Omega}|\nabla u|^{p-2} \nabla u \cdot \nabla(v-u) d x+\int_{\Omega} a(x)|u|^{p-2} u(v-u) d x \\
& -\int_{\Omega} f(x, u)(v-u) d x \\
= & -\|u\|_{0}^{p}+\int_{\Omega}|\nabla u|^{p-2} \nabla u \cdot \nabla v d x+\int_{\Omega} a(x)|u|^{p-2} u v d x \\
& +\int_{\Omega} f(x, u) u d x-\int_{\Omega} f(x, u) v d x \\
\leq & -\|u\|_{0}^{p}+c_{1}\|u\|_{0}^{p-1}+\int_{\Omega}\left[g(x)|u|^{\alpha+1}+h(x)|u|\right] d x \\
& +\int_{\Omega}\left[g(x)|u|^{\alpha}|v|+h(x)|v|\right] d x \\
\leq & -\|u\|_{0}^{p}+c_{1}\|u\|_{0}^{p-1}+c_{2}\|u\|_{0}^{\alpha+1}+c_{3}\|u\|_{0}+c_{4}
\end{aligned}
$$

for each $u \in W^{1, p}(\Omega)$, where $c_{1}, c_{2}, c_{3}, c_{4}$ are positive constants. This implies that $\left\langle\varphi_{2}^{\prime}(u), v-u\right\rangle \rightarrow-\infty$ as $\|u\|_{0} \rightarrow \infty$. Hence there exists a positive number $r>0$ such that $\left\langle\varphi_{2}^{\prime}(u), v-u\right\rangle<0$ as $\|u\|>r$. This shows that $P(v)$ is a bounded subset of $W^{1, p}(\Omega)$. Note that $\varphi_{2}$ is continuously differentiable so that $P(v)$ is closed, obviously. Hence $P(v)$ is a weakly compact subset of $W^{1, p}(\Omega)$.

By virtue of FKKM theorem (see [5]) $\bigcap_{v \in W^{1, p}(\Omega)} P(v) \neq \varnothing$. Hence there exists a function $u \in W^{1, p}(\Omega)$ such that

$$
\left\langle\varphi_{2}^{\prime}(u), v-u\right\rangle \geq 0
$$


for all $v \in W^{1, p}(\Omega)$. This implies that $u$ is a critical point of $\varphi_{2}$. Therefore, $u$ is a solution of problem (2.1).

By using a similar method we can prove following Theorem 4 .

Theorem 4. If there exist $\alpha \in[0, p-1), g \in L^{\frac{p}{p-\alpha-1}}\left(\Omega, R^{+}\right)$and $h \in L^{q}\left(\Omega, R^{+}\right)$ $\left(q=\frac{p}{p-1}\right)$ such that

$$
|f(x, t)| \leq g(x)|t|^{\alpha}+h(x)
$$

for every $t \in R$ and a.e. $x \in \Omega$, then problem (1.1) has at least a solution in $W_{0}^{1, p}(\Omega)$.

Theorem 5. Assume that $p>N$ and $f=f_{1}+f_{2}$ is such that $f_{i}: \Omega \times R \rightarrow R$ is a Carathéodory function $(i=1,2)$, and for each $s>0$, $\sup _{|t|<s}\left|f_{1}(x, t)\right| \in L^{1}(\Omega)$. If the following conditions hold:

(i) there exist $g, h \in L^{1}(\Omega, R), M>0$ and $\alpha \in[0, p-1)$ such that

$$
\frac{f_{1}(x, t)}{t} \leq \frac{g(x)|t|^{\alpha-1} t+h(x)}{t}
$$

for all $|t| \geq M$ and a.e. $x \in \Omega$,

(ii)

$$
\sup _{\xi \in R} F_{1}(x, \xi) \leq 0
$$

a.e. $x \in \Omega$ and there exist two numbers $r_{0}>0$ and $\xi_{0} \in R$ such that

$$
\frac{\left|\xi_{0}\right|^{p} \int_{\Omega} a(x) d x}{p}-\int_{\Omega} F_{1}\left(x, \xi_{0}\right) d x<r_{0}
$$

and

$$
F_{2}\left(x, \xi_{0}\right)=\sup _{|\xi| \leq k\left(p r_{0}\right)^{\frac{1}{p}}} F_{2}(x, \xi)
$$

for a.e. $x \in \Omega$, where

$$
k=\sup _{u \in W^{1, p}(\Omega) \backslash\{0\}} \frac{\|u\|_{\infty}}{\|u\|_{0}},
$$

then problem (2.1) admits at least one solution.

Proof. Let

$$
\Psi(u)=\frac{\|u\|_{0}^{p}}{p}-\int_{\Omega} F_{1}(x, u) d x, \Phi(u)=-\int_{\Omega} F_{2}(x, u) d x
$$

for all $u \in W^{1, p}(\Omega)$, and let

$$
\varphi(r)=\inf _{u \in \Psi^{-1}((-\infty, r))} \frac{\Phi(u)-\inf _{c l_{w}\left(\Psi^{-1}((-\infty, r))\right)} \Phi(v)}{r-\Psi(u)},
$$

where $c l_{w}\left(\Psi^{-1}((-\infty, r))\right)$ denotes the weakly closure of the set $\Psi^{-1}((-\infty, r))$. Since $p>N$, the embedding operator $i: W^{1, p}(\Omega) \hookrightarrow C^{0}(\Omega)$ is compact. Hence $\Phi$ and $\Psi$ are weakly lower semicontinuous and Gateaux differentiable in $W^{1, p}(\Omega)$. From the proof of Theorem 1 we know that $\Psi$ is coercive. For each 
$v \in c l_{w}\left(\Psi^{-1}\left(\left(-\infty, r_{0}\right)\right)\right)$ there exists a sequence $\left\{v_{m}\right\} \subset \Psi^{-1}\left(\left(-\infty, r_{0}\right)\right)$ such that $v_{m} \rightarrow v$. By $\Psi\left(v_{m}\right) \leq r_{0},\left\|v_{m}\right\|^{p} \leq p r_{0}+\int_{\Omega} F_{1}\left(x, v_{m}\right) d x \leq p r_{0}$ and hence, $\|v\|^{p} \leq p r_{0}$. Hence

$$
\varphi\left(r_{0}\right) \leq \inf _{u \in \Psi^{-1}\left(\left(-\infty, r_{0}\right)\right)} \frac{\Phi(u)-\inf _{\|v\|^{p} \leq p r_{0}} \Phi(v)}{r_{0}-\Psi(u)} .
$$

Let $w_{0}(x) \equiv \xi_{0}$ for all $x \in \Omega$. Then $\Psi\left(w_{0}\right)=\frac{\int_{\Omega} a(x)\left|\xi_{0}\right|^{p} d x}{p}-\int_{\Omega} F_{1}\left(x, \xi_{0}\right) d x<r_{0}$. Moreover, for each $u \in W^{1, p}(\Omega)$ with $\|u\|^{p} \leq p r_{0}$, by (ii), one has $\Phi(u) \geq \Phi\left(w_{0}\right)$ and hence $\varphi\left(r_{0}\right)=0$. Note that $\inf _{u \in W^{1, p}(\Omega)} \Psi(u)=0$. By virtue of Theorem 2.5(a) in [6] $\Phi+\Psi$ has a critical point, and hence problem (2.1) has a solution.

In Theorem 5 , take $f_{1} \equiv 0$ or $f_{2} \equiv 0$, respectively. Then we have the following two interesting corollarys.

Corollary 6. Assume that $p>N$ and $f$ is such that $f: \Omega \times R \rightarrow R$ is a Carathéodory function. If there exist two numbers $r_{0}>0$ and $\xi_{0} \in R$ such that

$$
\frac{\left|\xi_{0}\right|^{p} \int_{\Omega} a(x) d x}{p}<r_{0}
$$

and

for a.e. $x \in \Omega$, where

$$
F\left(x, \xi_{0}\right)=\sup _{|\xi| \leq k\left(p r_{0}\right)^{\frac{1}{p}}} F(x, \xi)
$$

$$
k=\sup _{u \in W^{1, p}(\Omega) \backslash\{0\}} \frac{\|u\|_{\infty}}{\|u\|_{0}},
$$

then problem (2.1) admits at least one solution.

Corollary 7. Assume that $p>N$ and $f: \Omega \times R \rightarrow R$ is a Carathéodory function, and for each $s>0, \sup _{|t| \leq s}|f(x, t)| \in L^{1}(\Omega)$. If the following conditions hold:

(i) there exist $g, h \in L^{1}(\Omega, R), M>0$ and $\alpha \in[0, p-1)$ such that

$$
\frac{f(x, t)}{t} \leq \frac{g(x)|t|^{\alpha-1} t+h(x)}{t}
$$

for all $|t| \geq M$ and a.e. $x \in \Omega$,

$$
\sup _{\xi \in R} F(x, \xi) \leq 0
$$

a.e. $x \in \Omega$ and there exist two numbers $r_{0}>0$ and $\xi_{0} \in R$ such that

$$
\frac{\left|\xi_{0}\right|^{p} \int_{\Omega} a(x) d x}{p}-\int_{\Omega} F\left(x, \xi_{0}\right) d x<r_{0}
$$

for a.e. $x \in \Omega$, where

$$
k=\sup _{u \in W^{1, p}(\Omega) \backslash\{0\}} \frac{\|u\|_{\infty}}{\|u\|_{0}},
$$

then problem (2.1) admits at least one solution. 
Theorem 8. If there exist $g, h \in L^{1}(\Omega, R), M>0$, and $\alpha \in[0, p-1)$ such that

$$
\frac{f(x, t)}{t} \leq \frac{g(x)|t|^{\alpha-1} t+h(x)}{t}
$$

for all $|t| \geq M$ and a.e. $x \in \Omega, p>N$ and there exists a number $\delta>0$ such that, as $0<|t| \leq \delta$,

$$
\lambda_{1} V(x)|t|^{p-2} \leq \frac{f(x, t)}{t} \leq \bar{\lambda} V(x)|t|^{p-2},
$$

then problem (1.1) has at least two nontrivial solution in $W_{0}^{1, p}(\Omega)$.

Proof. Since $p>N$, the embedding operator $i: W_{0}^{1, p}(\Omega) \hookrightarrow C^{0}(\Omega)$ is compact. Hence there exists a number $c>0$ such that

$$
\|u\|_{\infty} \leq c\|u\|
$$

for all $u \in W_{0}^{1, p}(\Omega)$, and $\varphi_{1}$ is weakly lower semicontinuous on $W_{0}^{1, p}(\Omega)$.

For each $u \in E_{1}$, there exists a real number $k$ such that $u=k \varphi_{1}$, and as $\|u\| \leq \frac{\delta}{c}$

$$
\begin{aligned}
\varphi_{1}(u) & =\frac{1}{p}\|u\|^{p}-\int_{\Omega} F(x, u) d x \\
& =\frac{1}{p}|k|^{p}-\int_{\Omega}\left[\int_{0}^{1} f\left(x, k t \varphi_{1}\right) k \varphi_{1} d t\right] d x \\
& \leq \frac{1}{p}|k|^{p}-\int_{\Omega}\left[\int_{0}^{1} \lambda_{1} V(x) t^{p-1}|k|^{p} \varphi_{1}^{p} d t\right] d x \\
& =\frac{1}{p}|k|^{p}-\frac{1}{p}|k|^{p} \int_{\Omega} \lambda_{1} V(x) \varphi_{1}^{p} d x \\
& =0 .
\end{aligned}
$$

For each $u \in E_{2}$ with $\|u\| \leq \frac{\delta}{c}$,

$$
\begin{aligned}
\varphi_{1}(u) & =\frac{1}{p}\|u\|^{p}-\int_{\Omega} F(x, u) d x \\
& \geq \frac{1}{p}\|u\|^{p}-\int_{\Omega}\left[\int_{0}^{1} \bar{\lambda} V(x)|u|^{p} t^{p-1} d t\right] d x \\
& =\frac{1}{p}\|u\|^{p}-\frac{1}{p} \int_{\Omega} \bar{\lambda} V(x)|u|^{p} d x \\
& \geq 0 .
\end{aligned}
$$

Similar to Theorem 1 , we can prove that $\varphi_{1}$ is coercive on $W_{0}^{1, p}(\Omega)$, and hence $\varphi_{1}$ satisfies (PS) and is bounded below.

If $\inf \left\{\varphi_{1}(u): u \in W_{0}^{1, p}(\Omega)\right\}=0$, then all $u \in E_{1}$ with $\|u\| \leq \frac{\delta}{c}$ are minima of $\varphi_{1}$, which implies that $\varphi_{1}$ has infinite critical points. If $\inf \left\{\varphi_{1}(u): u \in W_{0}^{1, p}(\Omega)\right\}<$ 0 , then by Theorem 4 in [1] $\varphi_{1}$ has at least two nonzero critical points. Therefore, problem (1.1) has at least two nontrivial solutions in $W_{0}^{1, p}(\Omega)$. 
Remark. We need not assume $p>N$ in Theorem 3 and Theorem 4. But, there is the assumption $p>N$ in other theorems. The reason is that we need ensure $k=\sup _{u \in W^{1, p}(\Omega) \backslash\{0\}} \frac{\|u\|_{\infty}}{\|u\|_{0}}<+\infty$, which appear in our assumptions.

\title{
Acknowledgements
}

The authors would like to thank the referees for their careful reading of the paper. Their suggestions greatly improved the paper.

\section{References}

[1] G. Bonanno, P. Candito, Three solutions to a Neumann problem for elliptic equations involving the $p$-Laplacian, Arch. Math. 80 (2003), 424-29.

[2] G. Bonanno, R. Livrea, Multiplicity theorems for the Dirichlet problem involving the $p$-Laplacian, Nonlinear Analysis 54 (2003), 1-7.

[3] H. Brezis, Remarks on finding critical points, Comm. Pure Appl. Math. 44 (1991), 939-963.

[4] M. Cuesta, Eigenvalue problems for the $p$-Laplacian with indefinite weights, Electronic J. Differential Equations 33 (2001), Vol. 2001, 1-9.

[5] K. Fan, A generalization of Tychonoff' s fixed point theorem, Math. Ann. 142 (1961), 305-310.

[6] B. Ricceri, A general variational principle and some of its applications, J. Computa. Appl. Math. 113 (2000), 401-410.

\author{
Xian $\mathrm{Wu}$ \\ Department of Mathematics \\ Yunnan Normal University \\ Kunming Yunnan 650092 \\ P.R. China \\ e-mail: wuxian2001@yahoo.com.cn \\ Jilin Chen \\ Department of Mathematics \\ Zhaotong Teacher's College \\ Zhaotong Yunnan 65700 \\ P.R. China \\ e-mail: cj1611106@yahoo.com.cn
}

Received: 29 February 2008.

Revised: 20 July 2008.

Accepted: 2 September 2008. 